\title{
ИССЛЕДОВАНИЕ ПРОЯВЛЕНИЙ ПРЕДСЕРДНОЙ КАРДИОПАТИИ У ПАЦИЕНТОВ С СИСТЕМНОЙ ЭМБОЛИЕЙ БЕЗ УСТАНОВЛЕННОГО ИСТОЧНИКА
}

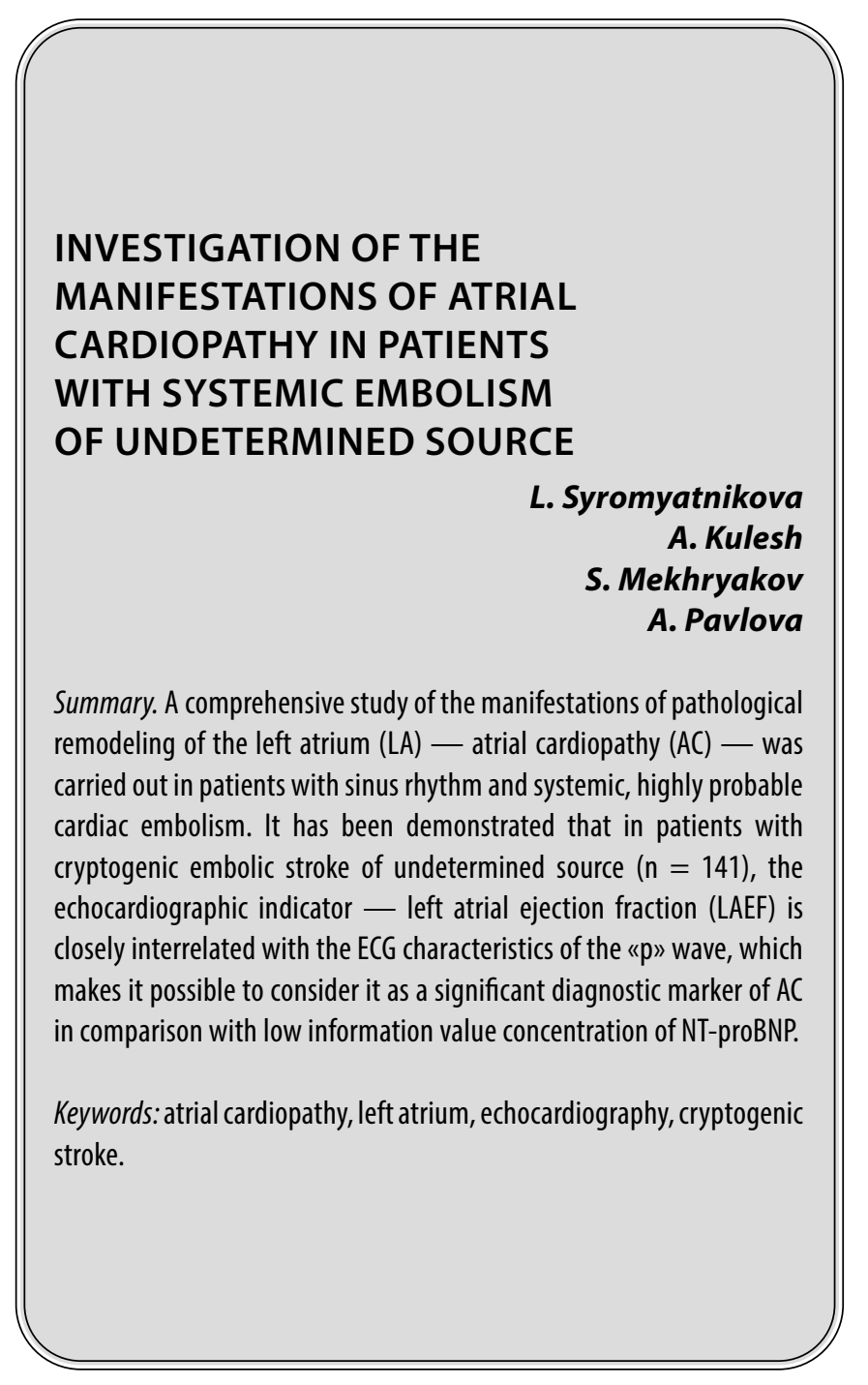

\section{Введение}

D 2021 году представлены результаты двух исследований LOOP [1] и STROKESTOP [2]. Их целью было исследование значения ранней диагностики фибрилляции предсердий (ФП) при помощи имплантируемого петлевого регистратора (Medtronic Reveal LINQ(TM) LOOP study) или ежедневной 2-х кратной регистрации электрокардиограммы (ЭКГ) в течение 2-х недель (STROKESTOP study) с последующим назначением антикоагулянтной терапии, оценкой ее влияния на жизненный прогноз. Оба исследования продемон-
Сыромятникова Людмила Илариевна

Д.м.н., профессор, ФГБОУ ВО «Пермский государственный медицинский университет имени академика Е.А. Вагнера» Минздрава России ilarievna@gmail.com

Кулеш Алексей Александрович

Д.м.н., профессор, ФГБОУ ВО «Пермский государственный медицинский университет имени академика Е.А. Вагнера» Минздрава России aleksey.kulesh@gmail.com

Мехряков Сергей Александрович Врач ультразвуковой диагностики, Региональный сосудистый чентр ГАУЗ ПК «Городская клиническая больница № 4» (Пермь)

heartolog@gmail.com

Павлова Анастасия Васильевна

Клинический ординатор, ФГБОУ ВО «Пермский государственный медицинский университет имени академика Е.А. Вагнера» Минздрава России anastasiya_pavlova_96@inbox.ru

Аннотация. Проведено комплексное исследование проявлений патологического ремоделирования левого предсердия (ЛП) - предсердной кардиопатии (ПК) - у пациентов с синусовом ритмом и системной, высоко вероятностной кардиальной эмболией. Продемонстрировано, что у пациентов с криптогенным инсультом с неустановленным источником эмболии ( $\mathrm{n=141)}$ эхокардиографический показатель — фракция опорожнения ЛП (LAEF) тесно взаимосвязан с ЭКГ характеристиками зубца «р», что позволяет его рассматривать как значимый диагностический маркер ПК в сравнении с малой информативностью концентрации NT-proBNP.

Ключевые слова: предсердная кардиопатия, левое предсердие, эхокардиография, криптогенный инсульт.

стрировали более высокую частоту регистрации ФП в группах вмешательства. Однако только в исследовании STROKESTOP назначение оральных антикоагулянтов (ОАК) определило небольшую, но статистически значимую клиническую выгоду в виде суммарного снижения количества случаев ишемического/геморрагического инсульта, системной эмболии, кровотечений, требующих госпитализации, а также общей смертности (HR0.96; 95\% Cl 0.92-1.00; P=0.045). В исследовании LOOP выявление 6-минутного эпизода ФП, независимо подтвержденного двумя кардиологами, не привело к улучшению прогноза данной группы больных. В ка- 
Таблица 1. Общая характеристика группы исследования пациентов с КИ (n=141)

\begin{tabular}{|l|l|}
\hline Показатель & Значение \\
\hline Возраст, лет & $64(55-70)$ \\
\hline Жен., абс. (\%) & $67(47,5)$ \\
\hline ИМТ, кг/м2 & $27,0(24-31)$ \\
\hline Артериальная гипертензия, абс. (\%) & $137(97,2)$ \\
\hline ИБС (стенокардия, ПИКС), абс. (\%) & $27(19,1)$ \\
\hline Сахарный диабет, абс. (\%) & $32(22,7)$ \\
\hline Статус курения, абс. (\%) & $40(28,4)$ \\
\hline OHMК в анамнезе, абс. (\%) & $44(31,2)$ \\
\hline NIHSS при поступлении, баллы & $6(3,5-8,5)$ \\
\hline NIHSS при выписке, баллы & $3(0-6)$ \\
\hline Шкала Рэнкина при выписке, баллы & $2(1-3)$ \\
\hline
\end{tabular}

Примечание: NIHSS - шкала инсульта национального института здоровья США, ИМТ — индекс массы тела, ОНМК - острое нарушение мозгового кровообращения, ИБС - ишемическая болезнь сердца.

честве одной из причин различий результатов рассматривается гипотеза, что в исследовании STROKESTOP при регистрации ЭКГ выявлялись клинически более значимые и продолжительные по времени эпизоды ФП, ассоциированные с более высоким риском развития инсульта. С нашей точки зрения решение вопроса персонализированного назначения ОАК у пациентов с факторами риска инсульта и субклинической ФП и/ или предсердных эпизодах высокой частоты [3] может быть осуществлено через понятие предсердной кардиопатии (ПК), определение критериев риска ее эмбологенности. Под ПК (кардиомиопатией, атриопатией) понимается патологическое состояние, когда под воздействием различных факторов (старение, фиброз, ишемия, воспаление, и т.д.) формируется электромеханическая дисфункция миокарда предсердий, нарастает прокоагулянтный потенциал, что ассоциируется с развитием системных эмболий, а также и ФП, что дополнительно усугубляет риск ишемических событий [4]. Наиболее подходящей моделью для оценки значимости ПК для развития системной эмболии является пациент с криптогенным инсультом (КИ) с неустановленным источником эмболии (ESUS). Признаки ПК наблюдаются в среднем у 65\% пациентов с КИ [5], из них в 35-45\% случаев ПК является источником эмболии при отсутствии данных за ФП.

\section{Цель работы}

Целью настоящей работы являлось определение особенностей ПК на модели пациентов с КИ при исключении иных причин формирования инсульта.

\section{Материалы и методы}

Обследован 141 пациент с ишемическим инсультом, в экстренном порядке поступивший в неврологическое отделение Регионального сосудистого центра ГАУЗ ПК «ГКБ № 4» Г. Перми. Проводилось выборочное включение пациентов, критериями включения в исследование явились: возраст от 18 до 75 лет, наличие верифицированного по данным нейровизуализации ишемического инсульта, подходящим под критерии ESUS. Так большие источники кардиоэмболии, которые могли бы послужить причиной инсульта (митральный стеноз, механические протезы, инфаркт миокарда в ближайшие 4 недели, аневризма левого желудочка и наличие тромбов в левых камерах сердца, фракция выброса менее 30\%) в рассматриваемой группе отсутствовали. В исследование не включались пациенты старше 75 лет; с догоспитальным результатом модифицированной шкалы Рэнкина (mRS)>3 баллов; с иными неврологическими, психиатрическими (в том числе с деменцией) и соматическими заболеваниями, определяющими тяжесть общего состояния; с осложненным течением инсульта; при наличии открытого овального окна. При поступлении регистрировалась ЭКГ в 12-отведениях, с последующей расширенной расшифровкой. При проведении трансторакальной эхокардиографии стандартный протокол дополнен показателями, позволяющими оценить анатомию и функцию ЛП. Всем пациентам определялся индексированный объем ЛП, а также проводилась оценка функциональных характеристик ЛП при помощи двух параметров - фракции опорожнения левого предсердия (LAEF) и функционального индекса левого предсердия (LAFI), рассчитываемых по формулам [6]. У части пациентов ( $\mathrm{n=43)}$ на 4-7-й день заболевания определена концентрация про-натрийуретического N-концевого пептида B-типа (NT-proBNP) с использованием стандартных тест-систем для иммуноферментного анализа крови (критерии нормы до 125 пг/мл).

Статистическая обработка проводилась с использованием пакета прикладных программ Statistica 10.0 
Таблица 2. Показатели ЭКГ при поступлении у пациентов с КИ.

\begin{tabular}{|c|c|}
\hline Показатель & Значение \\
\hline ЧСС в минуту & $68(58-76)$ \\
\hline RR max-RR min (c) & $0,04(0,02-0,04)$ \\
\hline Амплитуда P $\max ($ мм) & $1,1(1,0-1,5)$ \\
\hline Стандартное отведение (P max) (мм) & $2(2-2)$ \\
\hline Р стандартное отведение (с) & $0,08(0,08-0,1)$ \\
\hline Продолжительность $\mathrm{p} \vee_{1}$ (c) & $0,08(0,08-0,08)$ \\
\hline Амплитуда. p V1 (мм) & $1,0(1,0-1,1)$ \\
\hline Продолжительность терминальной части р (V1) (c) & $0,04(0,04-0,04)$ \\
\hline Амплитуда терминальной части p $\left(V_{I}\right)$, мм & $0,6(0,5-1,0)$ \\
\hline Терминальный индекс, мм* ${ }^{*}$ & $0,024(0,02-0,04)$ \\
\hline Продолжительность pQ (мс) & $165(150-183)$ \\
\hline Наличие патологического Q, абс. (\%) & $26(18,4)$ \\
\hline Продолжительность QRS (мс) & $92(86-104)$ \\
\hline Амплитуда Tmax (мм) & $4(2-5)$ \\
\hline QT (MC) & $404(386-430)$ \\
\hline QTC (MC) & $0,37(0,34-0,38)$ \\
\hline $\mathrm{RV}_{5}+\mathrm{SV}_{1}(\mathrm{MB})$ & $2,62(1,75-3,13)$ \\
\hline $\mathrm{R}_{a V L}+\mathrm{SV}_{3}(\mathrm{MB})$ & $17(12-20)$ \\
\hline$\left(\mathrm{R}_{a V L}+\mathrm{SV}_{5}\right)^{*} \mathrm{QRS}(\mathrm{MC})$ & $957(564-1318)$ \\
\hline Нарушение реполяризации, абс. (\%) & $38(26,9)$ \\
\hline АВ блокада, абс (\%) & $8(5,7)$ \\
\hline НБПНПГ, абс. (\%) & $7(5,0)$ \\
\hline БПНПГ, абс. (\%) & $5(3,5)$ \\
\hline БЛЗВПГ/БЛНВПГ, абс. (\%) & $2(1,4)$ \\
\hline БЛПВПГ, абс. (\%) & $9(6,4)$ \\
\hline ПБЛНПГ, абс. (\%) & $2(1,4)$ \\
\hline
\end{tabular}

(StatSoft Inc., США). Сравнительный анализ двух независимых групп по количественному признаку выполнялся с помощью критерия Манна-Уитни, по качественному признаку - с использованием критерия Х2. При проведении корреляционного анализа применялся критерий Спирмена. Средние величины в таблицах представлены как медиана и межквартильный интервал (Ме [25-й; 75-й перцентили]).

\section{Результаты \\ исслеАОвания}

Клинико-функциональная характеристика включенных в исследование $(n=141)$ представлена в таблице 1. Возраст пациентов составил 64 года, доля лиц женского пола была чуть менее 50\%. Отмечено наличие неблагоприятного метаболического статуса пациентов в виде избыточной массы тела/ожирения (33,3/27,7\%), сахарного диабета (23\%). Артериальная гипертензия имела облигатный характер и присутствовала в 97\% случаев. Важно отметить, что, несмотря на развитие повторного инсульта, у каждого третьего пациента, функциональный статус на момент выписки из стационара, опре- деленный по шкале Рэнкин, соответствовал медиане равной 2 баллам, у 75\% больных количество баллов не превышало 3 баллов.

Анализ ЭКГ, включал рутинную оценку амплитуды и продолжительности зубцов, нарушений ритма сердца и проводимости, критериев гипертрофии левого желудочка (ЛЖ), а также продолжительность и амплитуду зубца р, его терминальной части в отведении $\mathrm{V}_{l}$ (таблица 2). Данные характеристики зубца "p", а также терминальный индекс, рассчитываемый как произведение амплитуды и продолжительности терминальной части зубца $\mathrm{pV}_{l}$, в настоящее время рассматриваются как один из ЭКГ-критериев ПК [7]. Патологический зубец Q, как проявление перенесенного инфаркта миокарда выявлен в $18 \%$ случаев. Критерии гипертрофии миокарда левого желудочка Соколова-Лайона $\left(\mathrm{RV}_{5}+\mathrm{SV}_{l}(\mathrm{MB})>3,5\right.$ MB) соответствовали критериям нормы в 93,6\% случаев, согласно Корнельского произведения $\left(\left(\mathrm{R}_{a V L}+\mathrm{SV}_{5}\right)^{*} \mathrm{QRS}\right.$ $>2400$ мм* $^{*}$ М) - в 98,0\%, по Корнельскому вольтажному индексу, специфичному по полу - в 97,2\% случаев. Нарушение реполяризации характеризовало изменение полярности зубца Т у $27 \%$ пациентов. 
Таблица 3. Характеристика эхокардиографических показателей и концентрации NT-proBNP у пациентов с КИ.

\begin{tabular}{|l|l|}
\hline Показатель & 3начение \\
\hline Диаметр ЛП, см & $3,7(3,4-4,0)$ \\
\hline LAVI, мл/M² & $28,2(25,4-34,6)$ \\
\hline LAEF,\% & $54,5(51,8-56,5)$ \\
\hline LAFI, ед. & $0,37(0,31-0,44)$ \\
\hline NT-рroBNP, пг/мл & $151(24-308)$ \\
\hline
\end{tabular}

Примечание: ЛП - левое предсердие, LAVI - индекс объема левого предсердия, LAEF — фракция опорожнения левого предсердия, LAFI - функциональный индекс левого предсердия, NT-proBNP — про-натрийуретический N-концевой пептид B-типа.

Таблица 4. Характеристика пациентов группы КИ с учетом пороговых значений эхокардиографических показателей ПК

\begin{tabular}{|l|l|}
\hline Показатель & Значение \\
\hline Диаметр ЛП >4,15 см, абс. (\%) & $25(17,7)$ \\
\hline LAVI >36,3 мл/м², абс. (\%) & $16(11,3)$ \\
\hline LAEF >51,8\%, абс. (\%) & $106(75,2)$ \\
\hline LAFI $\leq 0,28$ ед., абс. (\%) & $27(19,1)$ \\
\hline
\end{tabular}

ЛП — левое предсердие, LAVI — индекс объема левого предсердия, LAEF — фракция опорожнения левого предсердия, LAFI - функциональный индекс левого предсердия.

Пациенты с КИ характеризовались морфологическими показателями ЛП, представленными в таблице 3, медианы всех эхокардиографических показателей, оценивающих ЛП, находились в пределах критериев нормы, также как и фракция выброса Лж (60\%).

Концентрация NT-proBNP у 56\% пациентов превышала критерии нормы (>125 пг/мл), варьируя в общей группе от 4 до 1695 пг/мл. Ранее нами установлено [8], что для диагностики ПК наибольшей специфичностью при низкой чувствительности обладает концентрация NT-proBNP, тогда как наиболее сбалансированным по чувствительности и специфичности эхокардиографическим показателем является LAEF. Порогом для LAEF установлено значение 51,8\%, для NT-proBNP 316 пг/мл. Эхокардиографические признаки ПК наблюдались в каждом четвертом или пятом случае (таблица 4). Концентрация NT-proBNP превышала пороговое значение для диагностики ПК в 23,3\% случаев ( $n=10)$.

Корреляционный анализ электрокардиографических показателей с эхокардиографическими маркерами ПК, значениями концентрации NT-proBNP (таблица 5) ожидаемо продемонстрировал наличие гемодинамических параллелей в виде прямой зависимости между чСС, диаметром и индексом объема ЛП, при снижении функционального индекса ЛП (LAFI) на фоне роста частоты сердечных сокращений. Увеличение таких параметров ЭКГ как амплитуда и продолжительность зубца «р» в стандартных отведениях и $\mathrm{V}_{l}$ отражают патологическое ремоделиро- вание ЛП в виде увеличения его диаметра при снижении фракции опорожнения. При этом именно показатель LAEF демонстрирует обратную взаимосвязь с характеристиками зубца «р» в грудном отведении $\mathrm{V}_{1}$, что вновь подтверждает его ценность в диагностике ПК. Чем ниже функциональная способность ЛП к опорожнению, тем выше амплитуда зубца «р», значение его терминального индекса. Интересно отметить, что уровень NT-proBNP продемонстрировал взаимосвязь с ЭКГ-критериями поражения ЛЖ, при отсутствии взаимосвязи с ЭКГ характеристиками ЛП. Выявлена прямая зависимость уровня NT-proBNP с продолжительностью желудочкового комплекса, признаками гипертрофии ЛЖ, нарушениями реполяризации. Данный результат указывает на малую информативность уровня NT-proBNP применительно к диагностике ПК.

\section{Обсужление}

ПК, как проявление патологического ремоделирования ЛП, может самостоятельно провоцировать эмболическое событие вследствие возникновения спектра предсердных аритмий, атриомегалии, фиброза, воспаления и нарушения свертывания [9]. В данной работе представлена характеристика и взаимосвязь ЭКГ и эхокардиографических маркеров ПК, концентрации NT-proBNP у пациентов с КИ. С учетом наличия статистически значимой отрицательной взаимосвязи между ЭКГ критериями гипертрофии, перегрузки предсердий и фракции опорожнения ЛП (LAEF), данный показатель может рас- 
Таблица 5. Результаты корреляционного анализа эхокардиографических маркеров ПК и концентрации NT-proBNP с показателями ЭКГ при поступлении.

\begin{tabular}{|c|c|c|c|c|c|}
\hline \multirow{2}{*}{ Показатель } & \multicolumn{5}{|l|}{ Значение } \\
\hline & Диаметр ЛП, см & LAVI, мл/M ${ }^{2}$ & LAEF, $\%$ & LAFI, ед. & NT-proBNP, пг/мл \\
\hline 4CC & NS & $0,22, p=0,026$ & NS & $-0,21, p=0,028$ & NS \\
\hline $\begin{array}{l}\text { Станд. отведение } \\
\text { (Р max) }\end{array}$ & $0,21, p=0,030$ & NS & $-0,27, p=0,006$ & NS & NS \\
\hline Р (с) станд. отведение & $0,19, p=0,048$ & NS & NS & NS & NS \\
\hline Амплитуда $\mathrm{p} \vee_{1}$ (мм) & $0,22, p=0,024$ & NS & $-0,21, p=0,028$ & NS & NS \\
\hline $\begin{array}{l}\text { Амплитуда } \\
\text { терминальной части } \mathrm{pV}_{I}\end{array}$ & NS & NS & $-0,29, p=0,003$ & NS & NS \\
\hline Терминальный индекс & NS & NS & $-0,33 p=0,005$ & $-0,29, p=0,02$ & NS \\
\hline $\begin{array}{l}\text { Продолжительность } \\
\text { pQ (c) }\end{array}$ & $0,24, p=0,012$ & $0,24, p=0,015$ & $-0,24, p=0,014$ & $-0,34, p<0,001$ & NS \\
\hline $\begin{array}{l}\text { Наличие } \\
\text { патологического Q, абс. } \\
\text { (\%) }\end{array}$ & NS & NS & NS & $-0,20, p=0,041$ & NS \\
\hline $\begin{array}{l}\text { Продолжительность } \\
\text { QRS (мс) }\end{array}$ & NS & NS & NS & NS & $0,33, p=0,044$ \\
\hline $\mathrm{RV}_{5}+\mathrm{SV}_{1}(\mathrm{MB})$ & NS & NS & NS & NS & $0,37, p=0,021$ \\
\hline $\mathrm{R}_{a V L}+\mathrm{SV}_{3}$ & NS & $0,32, p<0,001$ & NS & $-0,38, p<0,001$ & NS \\
\hline$\left(\mathrm{R}_{a V L}+\mathrm{SV}_{5}\right)^{*} \mathrm{QRS}(\mathrm{MC})$ & $0,24, p<0,001$ & $0,29, p=0,003$ & NS & $-0,32, p<0,001$ & NS \\
\hline $\begin{array}{l}\text { Нарушение } \\
\text { реполяризации, абс. (\%) }\end{array}$ & NS & NS & NS & NS & $0,41, p=0,026$ \\
\hline
\end{tabular}

сматриваться как наиболее значимый эхокардиографический параметр ранней диагностики ПК. В то время как концентрация NT-proBNP, вероятно, не может служит основой ранней диагностики дисфункции предсердий и требует замены на более чувствительные сывороточные маркеры ПК. Перспективными в этом отношении показателями являются среднерегионарный фрагмент предсердного натрийуретического пептида (MR-proANP), тканевый ингибитор матриксных металлопротеиназ 1 типа (TIMP-1) [10] и растворимый стимулирующий фактор роста, экспрессируемого геном 2 (sST2) [11].

\section{Зак^ючение}

Установление четких диагностических критериев ПК у больных с состоявшейся системной эмболией по- зволит сформировать целевую категорию пациентов для своевременного назначения антикоагулянтной терапии при отсутствии клинически подтвержденной ФП для улучшения их жизненного прогноза. С другой стороны, феномен ПК должен быть востребован и для совершенствования подходов первичной профилактики системных эмболий у лиц с высоким риском инсульта. Комплексная лабораторно-инструментальная оценка функции ЛП, выявление признаков его патологического ремоделирования позволит сформировать группу риска для продленного мониторинга ЭКГ, а при выявлении субклинической ФП и предсердных эпизодов высокой частоты - показания для назначения ОАК. Представляется вероятным, что данный подход позволит достоверно улучшить прогноз в группе вмешательства.

\section{ЛИТЕРАТУРА}

1. Svendsen JH, Diederichsen SZ, Højberg S et al. Implantable loop recorder detection of atrial fibrillation to prevent stroke (the LOOP study): a randomised controlled trial. Lancet. 2021; Epub ahead of print.

2. Svennberg E, Friberg L, Frykman V et al. Clinical outcomes in systematic screening for atrial fibrillation (STROKESTOP): a multicentre, parallel group, unmasked, randomised controlled trial. Lancet. 2021; Epub ahead of print.

3. ESC Guidelines for the diagnosis and management of atrial fibrillation developed in collaboration with the European Association of Cardio-Thoracic Surgery (EACTS). European Heart Journal 2020. 00.1-125.

4. Guichard JB, Nattel S. Atrial cardiomyopathy: a useful notion in cardiac disease management or a passing fad? // J. Am. Coll. Cardiol. 2017. Vol. 70, N6. P. 756765. 
5. Yaghi S, Bernstein RA, Passman R et al. Furie Cryptogenic Stroke: Research and Practice. Circ Res. 2017 Feb 3;120(3):527-40.

6. Sargento L, Vicente Simoes A, Longo S et al. Left atrial function index predicts long term survival in stable outpatients with systolic heart failure. Eur Heart J Cardiovasc Imaging. 2017 Feb;18(2):119-27.

7. Jalini S, Rajalingam R, Nisenbaum R et al. Atrial cardiopathy in patients with embolic strokes of unknown source and other stroke etiologies//Neurology. 2019. Vol. 92, N4. P. e288-e294.

8. Мехряков С.А., Кулеш А.А., Сыромятникова Л.И. и соавт. Биомаркеры предсердной кардиопатии у пациентов с разными патогенетическими подтипами ишемического инсульта. Неврология, нейропсихиатрия, психосоматика. 2020;12(6):33-41

9. Elkind MSV. Atrial cardiopathy and stroke prevention. Review. Curr Cardiol Rep. 2018 Sep 12;20(11):103.

10. Полянская Е.А., Веклич А.С., Козиолова Н.А. Новые диагностические критерии предсердной кардиомиопатии у больных сахарным диабетом 2 типа и фибрилляцией предсердий. Российский кардиологический журнал. 2021;26(3):4359.

11. Полянская Е.А., Козиолова Н.А. Поиск дополнительных диагностических критериев предсердной кардиомиопатии у больных с изолированной формой фибрилляции предсердий. Российский кардиологический журнал. 2021;26(1):4258.

( С сыромятникова Людмила Илариевна ( ilarievna@gmail.com ), Кулеш Алексей Александрович ( aleksey.kulesh@gmail.com ), Мехряков Сергей Александрович ( heartolog@gmail.com ), Павлова Анастасия Васильевна ( anastasiya_pavlova_96@inbox.ru ). Журнал «Современная наука: актуальные проблемы теории и практики»



